\title{
AGB stars of the intermediate-age LMC cluster NGC 1846
}

\section{Dredge up along the AGB}

\author{
T. Lebzelter ${ }^{1}$, M. T. Lederer ${ }^{1}$, S. Cristallo ${ }^{2}$, K. H. Hinkle ${ }^{3}$, O. Straniero ${ }^{2}$, and B. Aringer ${ }^{1,4}$ \\ 1 Department of Astronomy, University of Vienna, Tuerkenschanzstrasse 17, A1180 Vienna, Austria \\ e-mail: lebzelter@astro.univie.ac.at \\ 2 INAF, Osservatorio Astronomico di Collurania, 64100 Teramo, Italy \\ 3 National Optical Astronomy Observatories ${ }^{\star}$, PO Box 26732, Tucson, AZ 85726, USA \\ ${ }^{4}$ Dipartimento di Astronomia, Università di Padova, Vicolo dell'Osservatorio 3, 35122 Padova, Italy
}

Received 8 January 2008 / Accepted 21 April 2008

\section{ABSTRACT}

\begin{abstract}
Aims. We investigate the change in the surface abundance of ${ }^{12} \mathrm{C}$ during the evolution along the AGB, aiming to constrain third dredge-up models.

Methods. High-resolution, near-infrared spectra of a sample of AGB stars in the LMC cluster NGC 1846 were obtained. A cluster sample ensures a high level of homogeneity with respect to age, metallicity, and distance. The $\mathrm{C} / \mathrm{O}$ ratio and the ratio of ${ }^{12} \mathrm{C} /{ }^{13} \mathrm{C}$ were measured and compared with our evolutionary models.

Results. For the first time, we show the evolution of the $\mathrm{C} / \mathrm{O}$ and ${ }^{12} \mathrm{C} /{ }^{13} \mathrm{C}$ ratios along a cluster AGB. Our findings allow us to check the reliability of the evolutionary models and, in particular, the efficiency of the third dredge up. The increase in both $\mathrm{C} / \mathrm{O}$ and ${ }^{12} \mathrm{C} /{ }^{13} \mathrm{C}$ in the observed O-rich stars is reproduced by the models well. However, the low carbon isotopic ratios of the two C-stars in our sample indicate the late occurrence of moderate extra mixing. The extra mixing affects the most luminous AGB stars and is capable of increasing the abundance of ${ }^{13} \mathrm{C}$, while leaving unchanged the $\mathrm{C} / \mathrm{O}$ ratio, which has been fixed by the cumulative action of several third dredge-up episodes. We find indications that the $\mathrm{F}$ abundance also increases along the AGB, supporting an in situ production of this element.
\end{abstract}

Key words. stars: AGB and post-AGB - stars: variables: general - galaxies; star clusters

\section{Introduction}

To understand the role of low and intermediate mass stars within the cosmic matter cycle the efficiency of the third dredge up is a critical quantity. During the short but decisive asymptotic giant branch (AGB) phase the third dredge up is responsible for mixing the burning products and the material produced via the s-process to the surface from where it can be ejected into the interstellar medium by stellar mass loss (see e.g. Busso et al. 1999, for a review). Current stellar evolution models (e.g. Straniero et al. 1997; Herwig 2000; Busso et al. 2001; Stancliffe et al. 2004; Straniero et al. 2006; Karakas \& Lattanzio 2007) provide quite detailed predictions for the surface abundance changes during the AGB phase. Different models agree qualitatively. The dependency of dredge up on various parameters like mass and metallicity has been explored in the models (Iben \& Renzini 1983; Straniero et al. 2003). Quantitative checks of the model predictions for third dredge-up efficiency have been so far restricted to AGB stars in the solar neighborhood (e.g. Lebzelter \& Hron 2003; Busso et al. 2001) or to indirect methods like the study of the progeny of AGB stars or synthetic stellar evolution (e.g. van Eck et al. 2001; Marigo et al. 1999). However, for studying ongoing nucleosynthesis and the third dredge-up itself, observations of AGB stars are needed.

\footnotetext{
* Operated by the Association of Universities for Research in Astronomy, under cooperative agreement with the National Science Foundation.
}

Among the material dredged to the surface, ${ }^{12} \mathrm{C}$ is of special interest as mixing of this element changes an oxygen-rich star $(\mathrm{C} / \mathrm{O}<1)$ into a carbon-rich one $(\mathrm{C} / \mathrm{O}>1)$. This leads to significant changes in the chemistry and, as a result, in the atmospheric structure and the mass loss properties of the star. The value of the $\mathrm{C} / \mathrm{O}$ ratio is thus an indicator of the nucleosynthesis and mixing processes inside the star. For a few bright field AGB stars, measurements of the abundance of $\mathrm{C}$ and its isotopes exist (Lambert et al. 1986; Harris et al. 1987; Smith \& Lambert 1990). Values for C/O range between 0.25 and 1.6 (see Fig. 9 of Smith \& Lambert 1990).

Comparing the findings from field stars with nucleosynthesis and mixing models is hampered by the rather large uncertainty in luminosity and mass, two very critical parameters for the models. Clusters of stars offer an excellent possibility to investigate several questions of stellar astronomy. They provide samples of stars that are homogeneous in age and, in general, also in metallicity located at the same distance. Thus it is possible to determine their evolutionary status and their mass more accurately than for field stars. This provides an important advantage for comparison with models of stellar atmospheres and evolution.

To investigate highly evolved stars on the AGB, globular clusters seem the best choice due to the number of potential targets they include and due to the age range in which they are found. Globular clusters of the Milky Way are not good candidates when studying the effect of the third dredge up. They are, indeed, too old so that their present generation of AGB stars should have a very low envelope mass, too low for the 
occurrence of a substantial dredge up. The Magellanic Clouds, however, contain a population of intermediate age clusters (e.g. Girardi et al. 1995) with AGB stars in the mass range 1.5 to $2 M_{\odot}$.

In this paper we present measurements of the $\mathrm{C} / \mathrm{O}$ ratio and the isotopic ratio ${ }^{12} \mathrm{C} /{ }^{13} \mathrm{C}$ for a sample of AGB stars in the cluster NGC 1846. In a previous paper (Lebzelter \& Wood 2007), the variability of the AGB stars in this cluster was discussed. We refer to this paper for a recent overview of the literature on this cluster and will only repeat a short summary here, listing the values for the global parameters used in the present paper.

NGC 1846 is an intermediate age cluster belonging to the LMC. It has a metallicity of $[\mathrm{Fe} / \mathrm{H}]=-0.49$ (Grocholski et al. 2006). Lebzelter \& Wood (2007) have determined the mass of the AGB stars to be close to $1.8 M_{\odot}$ resulting in a cluster age of $1.4 \times 10^{9}$ years. A number of AGB stars have been identified by Lloyd Evans (1980), and we use his naming convention throughout this paper. Near infrared measurements and estimates of the bolometric magnitudes of the AGB stars have been presented by Frogel et al. (1990, and references therein). No stars with a high mid-infrared excess, an indicator of a very high mass loss rate, have been found (Tanabé et al. 1998; Lebzelter \& Wood 2007).

The cluster velocity has been determined by various authors in the past. Schommer et al. (1992) report a cluster velocity of $240 \mathrm{~km} \mathrm{~s}^{-1}$ derived from measurements of two individual stars (231 and $248 \mathrm{~km} \mathrm{~s}^{-1}$, respectively). This value coincides with the result by Freeman et al. (1983) and is in good agreement with the most recent study of individual stars in this cluster by Grocholski et al. (2006), who find a mean velocity of $235 \mathrm{~km} \mathrm{~s}^{-1}$ from their 17 cluster members.

\section{Observations and data reduction}

High-resolution, near-infrared spectra of 12 AGB stars in NGC 1846 from the list of Lloyd Evans (1980) were obtained with the Phoenix spectrograph (Hinkle et al. 1998) at Gemini South during six half-nights in December 2005. We observed all 12 targets in the $K$ band between 23620 and $23700 \AA$; for 10 of them, spectra were also taken in the $H$ band between 15540 and $15587 \AA$ A. Spectral resolution was set to approximately 50000 . For most of the targets we achieved an $\mathrm{S} / \mathrm{N}$ ratio of 65 or better. We obtained two to three observations per target and setting on different locations along the slit to allow for background subtraction. Total integration times between 45 and 90 min per target and wavelength range were used. The spectra of each star were extracted and co-added using IRAF.

Wavelength calibration in the $K$ band was done using telluric lines in the spectrum of an early type star that was also obtained. That spectrum was then used to correct the telluric features in the $K$ band spectra of the program stars using the IRAF task telluric. The wavelength range in the $H$ band was selected to be almost free of telluric lines to allow for a better comparison with synthetic spectra. Thus we used stellar $\mathrm{OH}$ lines present in the spectra of the O-rich sample stars for the wavelength calibration in the $H$ band. The $\mathrm{C}$-star spectra were then calibrated with the solution derived from the O-rich stars.

\section{Data analysis}

\subsection{Contents of the observed wavelength ranges}

The observed spectral ranges in the $H$ and $K$ bands are shown in Fig. 1 for the star LE8, i.e. for a representative of the O-rich case. Several spectral features are identified. The $H$ band range

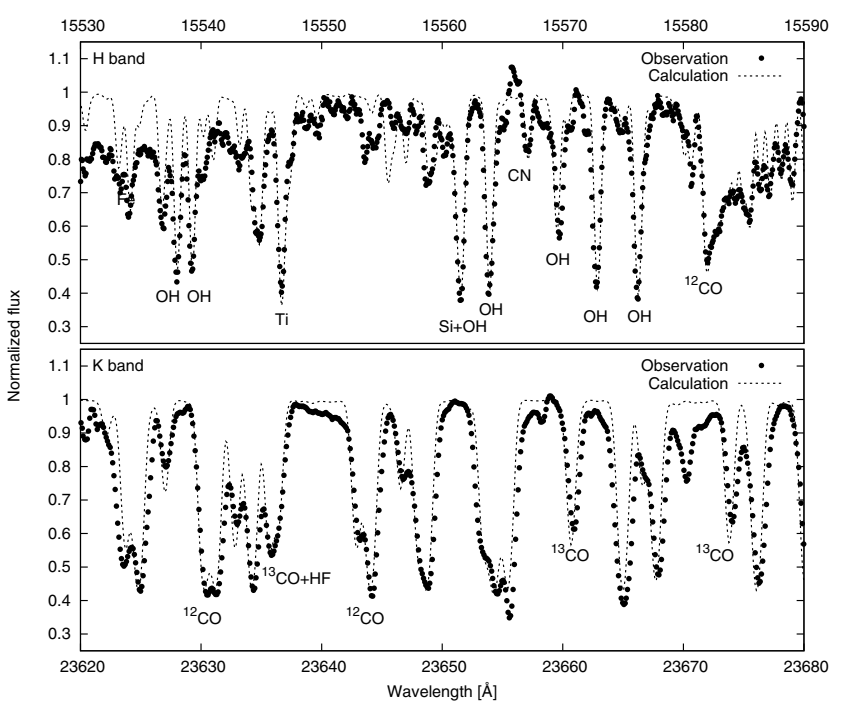

Fig. 1. Observed spectral ranges. Various features are identified. A fit to the spectrum with $T_{\text {eff }}=3550 \mathrm{~K}, \log g=0.25, \mathrm{C} / \mathrm{O}=0.30$ and a carbon isotopic ratio of 22 is given. In this fit, the $\mathrm{F}$ abundance has been reduced (see text). Due to the way the wavelength calibration was done (see text) the $H$ band spectrum is at rest wavelength while the $K$ band spectrum is shifted in wavelength according to the stellar velocity.

was selected to include a $\mathrm{CO}$ band head (3-0) and several lines of $\mathrm{OH}$. A few atomic lines can also be seen. Finally a number of $\mathrm{CN}$ lines are present within the observed wavelength range.

In the $\mathrm{C}$-rich case, the $H$-band spectra are completely dominated by lines of $\mathrm{CN}$ and $\mathrm{C}_{2}$. The $\mathrm{CO} 3-0$ band head is present, but its long wavelength side is strongly affected by neighboring features of the above-mentioned molecules. Lines of CO and CN are found in the observed wavelength range from the $K$ band.

\subsection{Synthetic spectra}

The synthetic spectra are based on model atmospheres that were calculated with the COMARCS code, a modified version of the MARCS code (Gustafsson et al. 1975; Jørgensen et al. 1992). The temperature and, accordingly, the pressure stratification of the models are derived under the assumption of a spherical configuration in hydrostatic and local thermodynamic equilibrium (LTE). The LTE implies chemical equilibrium that is evaluated in both the model calculation procedure and spectral synthesis, in either case taking all major opacity contributors into account such as $\mathrm{H}_{2} \mathrm{O}, \mathrm{TiO}, \mathrm{CO}$, and $\mathrm{CN}$ in the oxygen-rich case or $\mathrm{HCN}, \mathrm{C}_{2} \mathrm{H}_{2}, \mathrm{C}_{2}$, etc. in the carbon-rich case. For stars showing only small amplitude variability, LTE turns out to be a valid approximation, while potential uncertainties in the determination of abundances are due to a deviation from hydrostatic equilibrium rather than to non-LTE effects. COMARCS makes use of updated atomic and molecular opacities calculated with COMA (Aringer 2000). In calculating the model spectra, spherical radiative transfer routines originating from Windsteig et al. (1997) were used. The atomic line data were taken from VALD (Kupka et al. 2000), whereas the molecular data were compiled from several sources (see Cristallo et al. 2007, for an overview and Lederer \& Aringer 2007 for more recently added line lists). Model atmospheres and synthetic spectra that were calculated following the method described above have been shown to describe the spectra of cool giant stars appropriately (e.g. Loidl et al. 2001; Aringer et al. 2002). 
We want to point out that we calculated the model structure and the synthetic spectra in a fully consistent way with respect to spherical geometry and the atomic and molecular input data. Each atmospheric model is determined by six parameters: mass, metallicity, effective temperature, surface gravity, $\mathrm{C} / \mathrm{O}$ ratio, and microturbulent velocity. From the full set of parameters we kept the mass $\left(M=1.5 M_{\odot}\right)$ and the metallicity $([\mathrm{M} / \mathrm{H}]=-0.4)$ fixed (cf. next section for more details and references). The effective temperature was varied in steps of $50 \mathrm{~K}$, and the values for $T_{\text {eff }}$ range from $2600 \mathrm{~K}$ to $3850 \mathrm{~K}$ in our model set. In terms of the surface gravity, we chose a stepsize of 0.25 in logarithmic scale, i.e. $\log \left(g\left[\mathrm{~cm} \mathrm{~s}^{-2}\right]\right)$, ranging from -0.50 to +0.50 . The microturbulent velocity in the model calculations was set to $\xi=2.5 \mathrm{~km} \mathrm{~s}^{-1}$ apart from the model for the star LE13 where we adopted a value of $\xi=3.5 \mathrm{~km} \mathrm{~s}^{-1}$ (see below for details). The solar abundances were taken from Grevesse \& Noels (1993) whereby an inherent value of $\mathrm{C} / \mathrm{O}=0.48$ emerges. As for the variation in the $\mathrm{C} / \mathrm{O}$ ratio, we altered the abundance of carbon to arrive at the desired value and left the other abundances untouched. We changed the $\mathrm{C} / \mathrm{O}$ ratio in steps of 0.1 in general, but also reduced the stepsize to 0.05 when necessary for the fitting procedure (see next section).

The wavelength range covered in the calculation of the synthetic spectra was $6400-6450 \mathrm{~cm}^{-1}(15504-15625 \AA)$ in the $\mathrm{H}$ band and $4215-4270 \mathrm{~cm}^{-1}(23420-23725 \AA)$ in the $K$ band. The synthetic spectra were calculated with a resolution of 300000 and then convolved with a Gaussian to get a resolution of 50000 , which is about the value attained in our observed spectra. Subsequently, the macroturbulent velocity was accounted for by another convolution with a Gaussian. In the course of our analysis we also varied the carbon isotopic ratio ${ }^{12} \mathrm{C} /{ }^{13} \mathrm{C}$. The solar value as given in Anders \& Grevesse (1989) is about 89.9. The variation in the isotopic ratio has de facto no effect on the structure of the model atmosphere and thus was only considered in the spectral synthesis calculations. In the next section we give details in the variation of the above-mentioned parameters for the individual targets.

\subsection{Abundance determination}

To determine elemental abundances we used a two-step process. As a starting point we used $T_{\text {eff }}$ and $L$ values derived from broad-band, near-infrared photometry as described in Lebzelter \& Wood (2007). Assuming a stellar mass of $1.5 M_{\odot}$, we derived a starting value for the stellar radius and the surface gravity. Around these parameters we calculated a small grid of synthetic $H$ band spectra and selected the best fit with the observed spectrum both by visual inspection and by a $\chi^{2}$ approach. Several relative line strengths could be used to estimate the correctness of the fit. We varied $T_{\text {eff }}, \log g$, and $\mathrm{C} / \mathrm{O}$ ratio; among these three parameters, changes in $\log g$ had the weakest effect. Thus a small uncertainty in the mass or radius estimate of the star only has a minor influence on the derived abundances ${ }^{1}$. In the O-rich case, changes in $T_{\text {eff }}$ have the strongest effect on the $\mathrm{OH} / \mathrm{Fe}$ blend at $15570 \AA$, but an increase in temperature leads to a general increase in the strength of all features. An increase in $\mathrm{C} / \mathrm{O}$ makes the $\mathrm{CO}$ band head stronger and all the $\mathrm{OH}$ lines weaker at the same time. Additionally such a change leads to more prominent lines of $\mathrm{CN}$. In the chosen wavelength range, the model can be fitted to a well-defined pseudocontinuum. All these facts allow

\footnotetext{
1 We thus do also not have to bother about the small difference in mass between the one derived in Lebzelter \& Wood (2007) and the value assumed for calculating the synthetic spectra.
}

us to make good fits of the spectra in the O-rich case as illustrated in Fig. 1 and to derive a $\mathrm{C} / \mathrm{O}$ ratio from that. To achieve a good match of the lines, the macroturbulence was set to $3 \mathrm{~km} \mathrm{~s}^{-1}$. The influence of other values was tested. For the fit of LE16 and LE13, a macroturbulent velocity of 4 and $7 \mathrm{~km} \mathrm{~s}^{-1}$, respectively, was necessary to account for the broader lines observed in these stars. For LE13 we also increased the microturbulence to $3.5 \mathrm{~km} \mathrm{~s}^{-1}$ as mentioned before. We note that it was not our aim to measure the metallicity of the stars from our spectra because the selected wavelength ranges include only a small number of weak or strongly blended metal lines. The chosen metallicity of $[\mathrm{Fe} / \mathrm{H}]=-0.4$ for our models is close to the literature value and the metallic lines in the synthetic spectra are in reasonable agreement with the observed spectra. The fitting of the C-stars turned out to be much more complicated (see below).

In a second step, the results from the $H$ band spectrum were used as starting parameters for fitting the $K$ band spectrum. The latter is - especially for the O-rich case - rather insensitive to changes in $T_{\text {eff }}, \log g$, and C/O ratio. Thus these parameters cannot be determined from the $K$ band spectra alone. The chosen value of the macroturbulence could be confirmed. The main purpose for obtaining and analyzing the $K$ band spectra was to determine the ${ }^{12} \mathrm{C} /{ }^{13} \mathrm{C}$ isotopic ratio, which with the $\mathrm{C} / \mathrm{O}$ ratio is also a very reliable indicator of third dredge up. For this the O-rich spectra offer two (almost) unblended lines of ${ }^{13} \mathrm{CO}$ (2-0 R18 and 2-0 R19) and two blends including contributions from ${ }^{13} \mathrm{CO}$ lines. The best fit was selected by minimizing the squared difference in the spectral range around the two unblended ${ }^{13} \mathrm{CO}$ lines. Again, fitting of the $\mathrm{C}$-stars was more problematic and is briefly discussed separately below.

Uncertainties of the abundances were estimated from the range of parameters of the model spectra that still gave a good fit. For the $\mathrm{C} / \mathrm{O}$ ratio, the $T_{\text {eff }}$, and the $\log g$, value this was done from the fit of the $H$-band spectrum, for ${ }^{12} \mathrm{C} /{ }^{13} \mathrm{C}$ from the fit of the $K$-band spectrum. Because the carbon isotopic ratio has a negligible effect on the fit of the $H$-band spectrum, as verified by test calculations, the fitting procedure was not repeated with the determined ${ }^{12} \mathrm{C} /{ }^{13} \mathrm{C}$ ratio.

The $K$ band spectra also include a blend of the ${ }^{13} \mathrm{CO} 2-0 \mathrm{R} 21$ line with an HF line. When comparing this line blend with the synthetic spectrum using the isotopic ratio derived from the two unblended ${ }^{13} \mathrm{CO}$ lines, it turned out that this blend could not be fitted properly. An improvement in the fit can be either achieved by a change in $T_{\text {eff }}$ or by a modification of the $\mathrm{F}$ abundance. The first one was only possible in a very limited range due to the constraints set by the fit of the $H$ band spectrum. Thus we modified the abundance of $F$ until we achieved a good fit. This was only done for the O-rich stars because in the C-stars the blend is additionally affected by a nearby $\mathrm{CN}$ line.

The lowest excitation lines of $\mathrm{CO}$ in our $K$ band spectra could not be fitted well, especially in the case of the more luminous O-rich stars in our sample. An explanation for this could not be found; however, it may indicate a problem of the model structure in the outermost layers of the star, which may reflect dynamic effects or an outflow of material expected for variable stars.

\subsection{Carbon stars}

When fitting the C-rich stars, we were confronted with two major problems. The first one concerns the uncertainties of the line lists for the C-bearing molecules. The line lists of $\mathrm{CN}$ and $\mathrm{C}_{2}$, both critical for fitting the $H$-band spectrum, produced a number of features in the synthetic spectra that were obviously either 
at the wrong wavelength or of the wrong intensity. The $\mathrm{CN}$ list could be improved by replacing the wavelengths in the line list with the observed ones taken from Davis et al. (2005). This was possible for a number of strong lines, while the weak lines typically had no counterpart in the observed catalog. After that, a few $\mathrm{CN}$ lines with wrong line strengths still showed up in the synthetic spectrum. One of these lines, located at $6417.405 \mathrm{~cm}^{-1}$, was removed by hand from the $\mathrm{CN}$ list for the later calculation. This line is also not in the catalog of observed lines.

No observed reference is available for the $\mathrm{C}_{2}$ line list. Thus correcting this list is only possible by visual comparison of our spectra of C-stars in NGC 1846 with synthetic spectra. Within the studied range in the $H$-band, 5 lines were removed completely and 9 were shifted in wavelength to fit the observed spectra better. In this wavelength range, lines of the two molecules including a ${ }^{13} \mathrm{C}$ atom (i.e. ${ }^{13} \mathrm{C}^{12} \mathrm{C}$ and ${ }^{13} \mathrm{C}^{14} \mathrm{~N}$ ) play an important role for some of the blends. It turned out to be impossible to check the quality of these line data or to improve the line lists in that case because determining the carbon isotopic ratio was one of the aims of our project. We come back to this point in 4.3.

The second major problem was the absence of reliable "continuum" points in the C-star spectra. The main effect of decreasing $T_{\text {eff }}$ or increasing the $\mathrm{C} / \mathrm{O}$ ratio is the amount of depression of the (pseudo) continuum by a large number of weak and densely spaced lines over the whole spectral range. Thus the overall observed line depths can be fitted with a variety of combinations of these two parameters simply using different scaling factors between the observed and the calculated spectrum. At first glance, a possible point for the pseudocontinuum seems to be present close to $15571 \AA$ A. However, closer inspection reveals an absorption feature at that position in the observed spectrum that is missing in the synthetic spectrum. Therefore we think that this point is also not useable as a reference point. Thus we are limited to look for the comparably weak effect of using the ratios of features that seem to rely on one or the other of the two parameters. This, however, is hampered both by the uncertain line lists as discussed above and by the fact that a deeper $\mathrm{CN}$ or $\mathrm{C}_{2}$ line can either be achieved by an increase in $\mathrm{C} / \mathrm{O}$ or a decrease in temperature. For determining the $\mathrm{C} / \mathrm{O}$ ratio, we thus focused on the CO 3-0 band head that seems to be the only feature decoupled from this trend, but a larger uncertainty of the result compared to the O-rich case was unavoidable. The $\mathrm{CO}$ band head weakens relative to its neighboring features as $\mathrm{C} / \mathrm{O}$ is increased, and it is almost insensitive to temperature changes.

Fitting the $K$-band is eased by the lack of strong $\mathrm{C}_{2}$ lines, but as in the O-rich case, the depth of the various spectral features is rather insensitive to changes in the global parameters. Unfortunately, one of the two unblended ${ }^{13} \mathrm{CO}$ lines from the O-rich case is heavily blended by $\mathrm{CN}$ in the $\mathrm{C}$-rich case. That blending $\mathrm{CN}$ line seems to be real, as the shape of the two lines also differs in the observations, but the strength of the $\mathrm{CN}$ line is wrong in the synthetic spectrum (independent of changes in the global parameters). Thus this line could not be used and the determination of the isotopic ratio could be based only on one line with some additional indications from one blended feature in the C-rich case.

\subsection{Evolutionary models}

The theoretical models we use in the discussion of the observational results have been obtained by means of the FRANEC stellar evolutionary code (Chieffi et al. 1998). A detailed description of the input physics can be found in Straniero et al. (2006) and
Table 1. Radial velocities of NGC 1846 AGB stars. Concerning the membership of LE17 please see the discussion in the text.

\begin{tabular}{rrlr}
\hline \hline Star & $r v\left[\mathrm{~km} \mathrm{~s}^{-1}\right]$ & Star & $r v\left[\mathrm{~km} \mathrm{~s}^{-1}\right]$ \\
\hline LE1 & $251 \pm 2$ & LE11 & $244 \pm 3$ \\
LE2 & $248 \pm 2$ & LE12 & $247 \pm 2$ \\
LE3 & $248 \pm 3$ & LE13 & $242 \pm 2$ \\
LE6 & $247 \pm 2$ & LE17 & $262 \pm 1$ \\
LE8 & $250 \pm 1$ & H39 & $242 \pm 1$ \\
LE9 & $245 \pm 1$ & & \\
\hline
\end{tabular}

Cristallo et al. (2007). We calculated evolutionary sequences for various initial masses and compositions. In particular, models labeled as scaled solar, were obtained by scaling the solar abundances derived by Lodders (2003) of a factor $Z / Z_{\odot}$, where $Z$ is the assumed metallicity of the model. Then, $\alpha$-enhanced models were obtained by increasing the initial abundance of $\mathrm{O}, \mathrm{Ne}$, $\mathrm{Mg}, \mathrm{Si}, \mathrm{S}, \mathrm{Ca}$, and $\mathrm{Ti}$ by a common factor with respect to the corresponding scaled solar values (see Piersanti et al. 2007, for further details).

\section{Results}

\subsection{Cluster membership}

From the $K$-band spectra, we measured radial velocities of the target stars from individual lines of $\mathrm{CO}$. Results are presented in Table 1. For LE8, Olszewski et al. (1991) give a velocity of $248 \pm 5 \mathrm{~km} \mathrm{~s}^{-1}$, in excellent agreement with our value. The mean velocity of all investigated AGB stars in NGC 1846 is $248 \pm$ $5.5 \mathrm{~km} \mathrm{~s}^{-1}$, which is in good agreement with the published values for the cluster. The only star with a larger deviation from the mean velocity is LE17.

\subsection{O-rich stars}

The $\mathrm{C} / \mathrm{O}$ ratio and the ${ }^{12} \mathrm{C} /{ }^{13} \mathrm{C}$ ratio were determined for all six O-rich stars in our sample. The $\mathrm{C} / \mathrm{O}$ ratios between 0.2 and 0.65 were found with an uncertainty between \pm 0.05 and \pm 0.1 , and ${ }^{12} \mathrm{C} /{ }^{13} \mathrm{C}$ isotopic ratios between 12 and 60 were found. Temperatures of the best fit model spectra agree closely with the $T_{\text {eff }}$ values derived from near infrared photometry (Lebzelter \& Wood 2007). The result for each star is listed in Table 2 with the basic parameters of the stellar models used.

The C/O ratio obviously varies significantly from star to star, indicating different amounts of enriched material dredged up to the surface. It would be expected that an increased $\mathrm{C} / \mathrm{O}$ ratio due to the dredge up of ${ }^{12} \mathrm{C}$ would be accompanied by an increased isotopic abundance ratio. Indeed we find a nice correlation between $\mathrm{C} / \mathrm{O}$ and ${ }^{12} \mathrm{C} /{ }^{13} \mathrm{C}$ for the $\mathrm{O}$-rich stars as can be seen from Table 2. The values for $\mathrm{C} / \mathrm{O}$ and ${ }^{12} \mathrm{C} /{ }^{13} \mathrm{C}$ ratio found are in good agreement with findings from Smith \& Lambert (1990) for field stars. The minimum C/O ratio in our sample of 0.2 agrees closely with expectations from first dredge-up.

As described above, one blend including a line of $\mathrm{HF}$ (1-0 R7) is covered by our $K$ band observations. After limiting the various parameters affecting the shape and depth of this blend, namely mainly the temperature and the carbon isotopic ratio, from other parts of the stellar spectra we fitted the remaining difference between the observed and the calculated blend profile by a change in the $\mathrm{F}$ abundance. Due to the rather large uncertainties of the $\mathrm{C}$-star fitting we decided to derive the $\mathrm{F}$ abundance only for the O-rich stars. 
Table 2. Resulting abundances and model fit parameters for the sample stars. The last two columns give the periods and luminosities taken from Lebzelter \& Wood (2007).

\begin{tabular}{lcccccccc}
\hline \hline Star & $\mathrm{C} / \mathrm{O}$ & ${ }^{12} \mathrm{C} /{ }^{13} \mathrm{C}$ & {$[\mathrm{F} / \mathrm{Fe}]$} & $T_{\text {eff }}$ & $\log g$ & $\begin{array}{c}\text { Macroturbulent } \\
\text { Velocity }\end{array}$ & $P[\mathrm{~d}]$ & $\log L / L_{\odot}$ \\
\hline LE2 & $1.9 \pm 0.2$ & $65 \pm 15$ & - & $2600 \mathrm{~K}$ & -0.25 & 10 & $150 / 289$ & 3.835 \\
LE8 & $0.3 \pm 0.05$ & $20 \pm 2$ & -0.35 & $3550 \mathrm{~K}$ & 0.25 & 3 & 51 & 3.719 \\
LE9 & $0.2 \pm 0.05$ & $13 \pm 2$ & -0.42 & $3650 \mathrm{~K}$ & 0.25 & 3 & - & 3.604 \\
LE11 & $1.7 \pm 0.2$ & $60 \pm 10$ & - & $2900 \mathrm{~K}$ & -0.25 & 10 & $227 / 1066$ & 3.842 \\
LE13 & $0.65 \pm 0.1$ & $60 \pm 5$ & +0.40 & $3600 \mathrm{~K}$ & 0.0 & 7 & $92 / 837$ & 3.806 \\
LE16 & $0.44 \pm 0.05$ & $43 \pm 2$ & -0.20 & $3600 \mathrm{~K}$ & 0.0 & 4 & 57 & 3.645 \\
LE17 & $0.44 \pm 0.05$ & $30 \pm 4$ & +0.08 & $3700 \mathrm{~K}$ & 0.0 & 3 & 61 & 3.491 \\
H39 & $0.2 \pm 0.05$ & $12 \pm 2$ & -0.71 & $3650 \mathrm{~K}$ & 0.25 & 3 & 33 & 3.538 \\
\hline
\end{tabular}

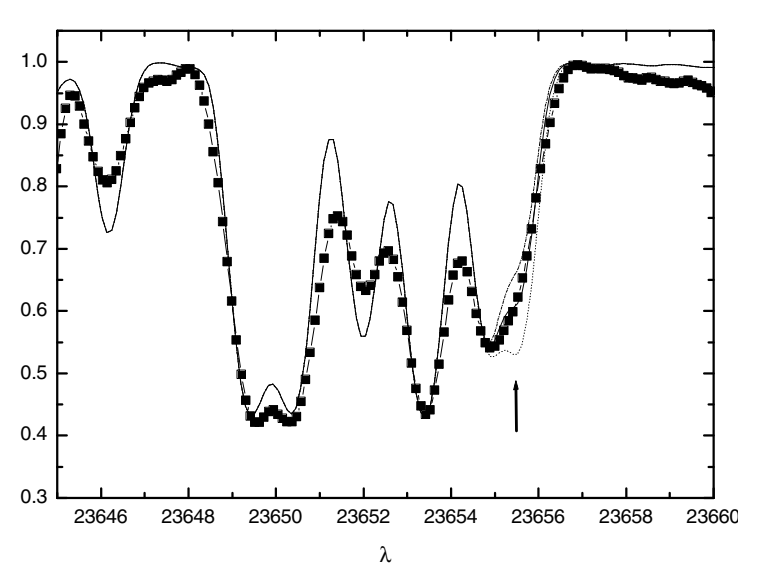

Fig. 2. Part of the $K$ band spectrum of LE8. The location of the HF line is marked by an arrow. Beside the spectrum (line + symbol) three models are plotted with a variable $\mathrm{F}$ abundance of -0.1 dex (dotted), $-0.5 \mathrm{dex}$ (solid), and $-0.7 \mathrm{dex}$ (dash-dot) relative to a solar value $(-7.44)$ and scaled for the average cluster metallicity, respectively.

Figure 2 shows as an example part of the spectrum of LE8 including the HF line (marked by an arrow). Overplotted are three models with different $\mathrm{F}$ abundances. Obviously, Fluorine is underabundant in LE8 relative to the other metals. It turns out that the $\mathrm{F}$ abundance has to be changed from star to star to allow for a reasonable fit of the observed blend. Uncertainty in determining the $\mathrm{F}$ abundance comes from both the temperature and the ${ }^{12} \mathrm{C} /{ }^{13} \mathrm{C}$ ratio (via a ${ }^{13} \mathrm{CO}$ line in the blend). We made an estimate of the uncertainty by varying these two parameters around the values determined from other parts of the spectra and then summing in quadrature the respective uncertainties. Taking into account this uncertainty and the fact that we have only one HF line to derive the abundance the results of course have to be taken with some caution. Confirmation from other HF lines is needed to limit the possible effect of unidentified lines on the profile of the blend and the derived abundances.

\subsection{C-rich stars}

The C/O ratio was derived for two C-rich stars, LE11 and LE2. For the other C-rich stars, either no $H$ band spectra were available (as a result of limited observing time) or no acceptable fit of the spectrum could be achieved. For the latter case, the starting influence of stellar variability on the stellar spectrum may play some role besides the above-mentioned problems.

The derived $T_{\text {eff }}$ and C/O for the C-rich star LE11 come with a much larger uncertainty. From the strength of the CO 3-0 band head, we exclude a $\mathrm{C} / \mathrm{O}$ of less than 1.4. A macroturbulent

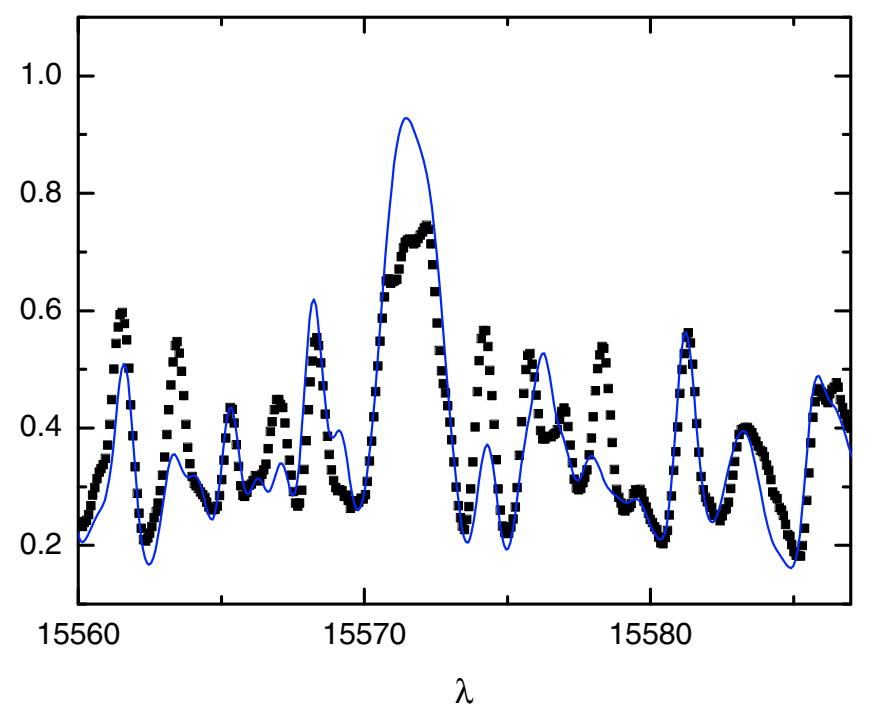

Fig. 3. Spectrum of the carbon star LE11 compared to a model fit with $\mathrm{C} / \mathrm{O}=1.7$ and $T_{\text {eff }}=2950 \mathrm{~K}$. See text for details.

velocity of $10 \mathrm{~km} \mathrm{~s}^{-1}$ is needed to both fit the width of the features and their strength. The temperature can be constrained even less. If we use the value from near infrared photometry, we get a $T_{\text {eff }}$ of $2950 \mathrm{~K}$. As noted by Lebzelter \& Wood (2007), this temperature is likely to be too low as pulsation properties instead suggest a value close to $3150 \mathrm{~K}$. We produced model spectra around both temperatures. A value of $T_{\text {eff }}=2950 \mathrm{~K}$ seems to give the better fit, but the sensitivity of the synthetic spectrum on this parameter is not very high.

Concerning an upper limit of the $\mathrm{C} / \mathrm{O}$ ratio an overall fit of the spectrum of similar quality can be reached for various combinations of $\mathrm{C} / \mathrm{O}$ and $T_{\text {eff }}$ with a higher temperature requiring a higher $\mathrm{C} / \mathrm{O}$ ratio. As a rule of thumb an increase in $T_{\text {eff }}$ by $100 \mathrm{~K}$ can be compensated by an increase of C/O by 0.1 in the parameter region under consideration. Taking the uncertainties of the line lists into account we cannot exclude a $\mathrm{C} / \mathrm{O}$ ratio as high as 2.0, but an upper limit of 1.9 seems reasonable due to constraints set by the range of possible temperatures defined by the $K$ band spectrum, the near infrared color and the pulsational behavior, respectively. In Fig. 3 we show a fit with $\mathrm{C} / \mathrm{O}=1.7$ and $T_{\text {eff }}$ of $2950 \mathrm{~K}$.

With this value we derived a ${ }^{12} \mathrm{C} /{ }^{13} \mathrm{C}$ ratio of about 60 from the $K$ band spectrum. The derived isotopic ratio depends on the temperature, the $\mathrm{C} / \mathrm{O}$ ratio, and the selection of the point in the synthetic spectrum to which the observed one is scaled. The $\mathrm{C} / \mathrm{O}$ ratio has the least influence and, within the constraints set by the $H$ band spectrum, its uncertainty can be neglected. The 


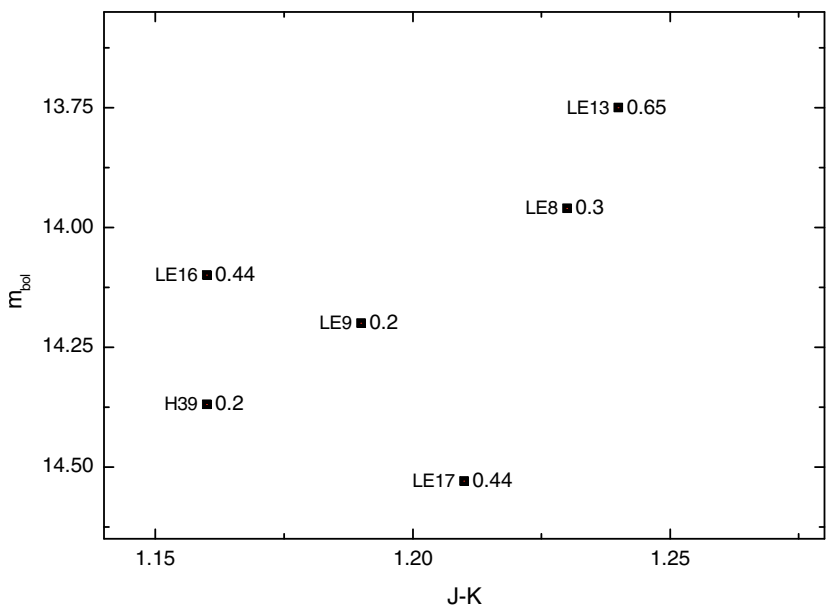

Fig. 4. Color-magnitude diagram of the O-rich stars in our sample. The $\mathrm{C} / \mathrm{O}$ ratio of each data point is given.

temperature is an important parameter for $T_{\text {eff }}$ above $2900 \mathrm{~K}$. Below that value, uncertainties in this parameter affect the resulting ${ }^{12} \mathrm{C} /{ }^{13} \mathrm{C}$ ratio less and less. The selection of the "pseudocontinuum" is of high importance in this low temperature regime. From a comparison between the observed spectra and synthetic spectra by varying the mentioned parameters, we derive a possible range for the isotopic ratio of \pm 10 around the mean value.

Additionally we were able to produce a reasonable fit for the spectra of LE2 when using a temperature between 2600 and $2850 \mathrm{~K}$, the $H$ band spectrum indicates a $\mathrm{C} / \mathrm{O}$ ratio of $1.9 \pm 0.2$. The lower temperature nicely corresponds to the one derived from the $J-K$ value (Lebzelter \& Wood 2007). The resulting ${ }^{12} \mathrm{C} /{ }^{13} \mathrm{C}$ ratio is close to $65 \pm 15$.

While we were not able to make a good fit of the $H$ and $K$ band spectra of LE1, we made a qualitative comparison of LE1 and LE2. Both wavelength ranges indicate that LE1 has a higher $\mathrm{C} / \mathrm{O}$ ratio than LE2. Unfortunately, a quantitative estimate was not possible.

\section{Discussion}

\subsection{Dredge up along the $A G B$}

In Fig. 4 the location of the O-rich stars of our sample in a colormagnitude diagram are shown. The data points are labeled with their respective $\mathrm{C} / \mathrm{O}$ ratio. Color and $m_{\text {bol }}$ values used here are taken from Lebzelter \& Wood (2007). As shown in that paper, color variations of these stars are very small and can be neglected in the present discussion. The $m_{\text {bol }}$ values are derived from the near infrared magnitudes using the transforms in Houdashelt et al. (2000a,b). For details we refer to Lebzelter \& Wood (2007). The sample stars cover a significant range in brightness along the AGB, thus allowing us to look for changes in the $\mathrm{C} / \mathrm{O}$ and ${ }^{12} \mathrm{C} /{ }^{13} \mathrm{C}$ ratios with increasing luminosity. We can exclude that these stars belong to the RGB. Indeed, the RGB tip observed by Lebzelter \& Wood (2007) is definitely fainter than the faintest star in the sample, LE17. This occurrence is confirmed by our evolutionary models. Actually, LE17 is only slightly brighter than the RGB tip of the $1.5 M_{\odot}$ model, but its ${ }^{12} \mathrm{C} /{ }^{13} \mathrm{C}$ ratio (30) is definitely greater than expected for the brightest RGB stars (see e.g. Sneden 1991, Gratton et al. 2000). LE13, the brightest star in our sample, is also the brightest O-rich AGB star known in this cluster. However, the resulting picture is far from clear. Indeed we find the star with the highest $\mathrm{C} / \mathrm{O}$ value at the top. But the two stars with a C/O ratio close to 0.44 (LE16 and LE17) do not seem to fit into a steady increase with luminosity at all. They also seem to be offset from the other stars, which seem to form some sequence in the CMD.

We note here that the luminosity at which an AGB star is observed does not necessarily have to be representative of its evolutionary state along the AGB. During thermal pulses the brightness can change significantly both to lower and to higher values. It was suggested in Lebzelter \& Wood (2007) that at least the atypically low luminosity of two of the carbon stars in this cluster can be explained by a deviation from the mean interpulse luminosity during a thermal pulse.

LE17 seems to be an outlier in various aspects. Assuming that the star is a cluster member, its relatively low luminosity, coupled to its relatively large $J-K$, could be explained if this star is undergoing a post-flash dip phase (Iben \& Renzini 1983). The expansion powered by a thermal pulse indeed causes a significant decrease in the luminosity and in the effective temperature. From our models, we derive that variations up to $1 \mathrm{mag}$ and $200 \mathrm{~K}$ (in $M_{\mathrm{bol}}$ and $T_{\mathrm{eff}}$, respectively) have to be expected. Alternative scenarios for explaining the characteristics of LE17 could be either an unresolved binary or a long secondary period similar to the ones already found in several AGB stars (e.g. Wood et al. 2004). The observed offset in velocity of LE17 from the other AGB stars in our sample could be consistent with both of these scenarios. The star's obvious enrichment in ${ }^{12} \mathrm{C}$, however, could be best explained by the post-flash dip scenario.

An alternative would be that LE17 is not a cluster member but a background star. Indeed this solution cannot be ruled out. Frogel et al. (1990) list the star as a cluster member based on its projected distance to the cluster center. Lebzelter \& Wood (2007) used the location of the star in the color-magnitude diagram as an argument in favor of the cluster membership. On the other hand, the radial velocity of the star is offset from the other AGB stars measured by almost $20 \mathrm{~km} \mathrm{~s}^{-1}$. In their work on the velocity dispersion in LMC clusters Grocholski et al. (2006) chose a range in velocity of $\pm 10 \mathrm{~km} \mathrm{~s}^{-1}$ around the mean cluster velocity to separate members from non-members. According to this rule LE17 would not be a member. Based on the present data, it is difficult to distinguish between the two possibilities.

Concerning the other "anomalous" star, LE16, one could speculate that the observed AGB stars may belong to two sequences, one including the stars with $\mathrm{C} / \mathrm{O}$ ratios between 0.2 and 0.3 , and another one, shifted to the blue, with the two remaining stars. We come back to this point below.

As mentioned above there is a qualitative agreement in the derived abundances between the increase in $\mathrm{C} / \mathrm{O}$ and ${ }^{12} \mathrm{C} /{ }^{13} \mathrm{C}$. Thus, a wrong abundance for the two deviating stars is not very likely. Note that all the stars with $\mathrm{C} / \mathrm{O}>0.2$ and ${ }^{12} \mathrm{C} /{ }^{13} \mathrm{C}>$ 15 but LE17 are brighter than the minimum luminosity for the occurrence of the third dredge up, as predicted by our models. Taking the mean luminosity of the interpulse period just after the occurrence of the first TDU episode, we find that all stars with $m_{\text {bol }}$ brighter than $14.1 \mathrm{mag}$ should be ${ }^{12} \mathrm{C}$ enhanced. Once again, the anomaly of LE17 could be solved if the star was in the post-flash dip.

We illustrate the relation between $\mathrm{C} / \mathrm{O}$ and ${ }^{12} \mathrm{C} /{ }^{13} \mathrm{C}$ in Fig. 5 . While there is some scatter, a relation between these two quantities can clearly be seen. In the same figure, we also plotted the expected relations from evolutionary models. The solid line represents our reference model: $M=1.9 M_{\odot}$ (1.8 at the beginning of the TP-AGB phase), $Z=0.006,[\alpha / \mathrm{Fe}]=0.2, Y=0.27$. The mass has been chosen according to the pulsational mass derived by Lebzelter \& Wood (2007), while the moderate enhancement 


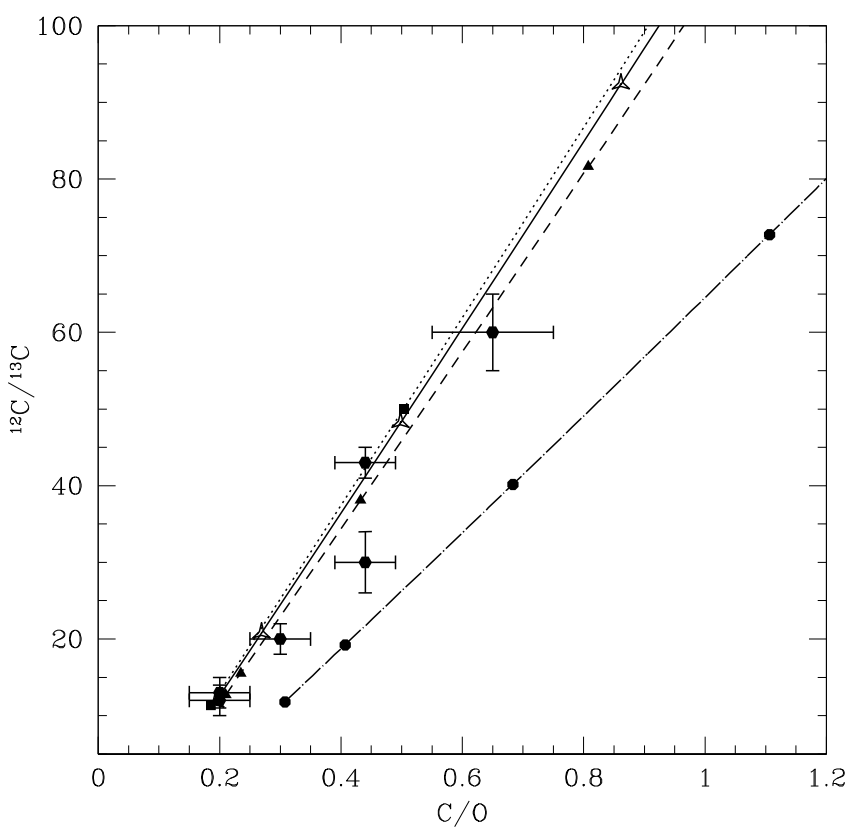

Fig. 5. $\mathrm{C} / \mathrm{O}$ versus ${ }^{12} \mathrm{C} /{ }^{13} \mathrm{C}$ for the $\mathrm{O}$-rich stars in our sample. For comparison predicted values from our evolutionary models are shown. The lines connect individual interpulse values for four sets of model parameters. See text for details.

of the $\alpha$ elements corresponds to the value generally claimed for the Large Magellanic Cloud (see e.g. Hill et al. 2000). The $\mathrm{C} / \mathrm{O}$ ratio of the two less evolved stars in our sample, $\mathrm{H} 39$ and LE9, supports such an assumption. Indeed, the expected $\mathrm{C} / \mathrm{O}$ ratio after the first dredge up is 0.35 , for a scaled solar composition, and 0.2 when $[\mathrm{O} / \mathrm{Fe}]=+0.2$. In this case, the total metallicity $(Z=0.006)$ would imply $[\mathrm{Fe} / \mathrm{H}]=-0.5$, in agreement with the latest spectroscopic determination for NGC 1846 (Grocholski et al. 2006). To evaluate the effects of possible variations of these model parameters, we also report the relations obtained by changing the mass $\left(1.5 M_{\odot}\right.$, dashed line), the total metallicity $(Z=0.003$, dotted line), and the initial abundance ratios (scaled solar instead of $\alpha$-enhanced, dot-dashed line).

It can be seen that all data points are found very close to the theoretical relation for the reference model, even if LE17 presents a lower carbon isotopic ratio (or too high $\mathrm{C} / \mathrm{O})^{2}$. Changes in initial mass and metallicity have little effect, so that the uncertainties of the present spectroscopic analysis do not allow us to distinguish among the different relations reported in Fig. 5. The model with a solar-scaled composition can instead be safely excluded. The boxes along the relations mark the interpulse values after each dredge up event. Close to these values we should have the highest probability of finding a star. In general, the agreement is remarkably good, with one exception (LE13).

Concerning the abundance of the light element F, we observe, as shown in Fig. 6, a change from an $\mathrm{F}$ underabundance to an $\mathrm{F}$ overabundance with increasing $\mathrm{C} / \mathrm{O}$ (relative to the solar value scaled to the iron abundance of the cluster). Accordingly, there is also a similar trend with the luminosity, as found for the $\mathrm{C} / \mathrm{O}$ ratio. Measurements of $\mathrm{HF}$ lines are a critical source for

\footnotetext{
2 Note that the abundances have been determined with a model atmosphere without $\mathrm{O}$ enhancement. To see if this has any effect on the derived ratios, we calculated a test model with $\mathrm{O}$ enhanced by +0.1 and +0.2 dex, respectively. It turned out that this enhancement only has a weak effect on the spectra which can be compensated for by small changes in the temperature. The $\mathrm{C} / \mathrm{O}$ and the ${ }^{12} \mathrm{C} /{ }^{13} \mathrm{C}$ ratios remain almost unchanged.
}

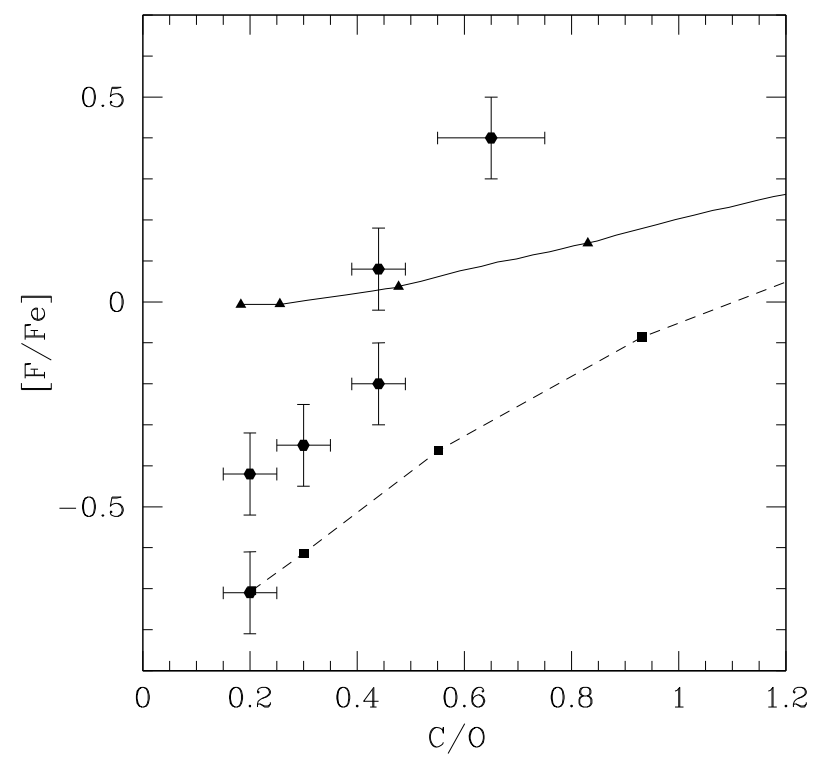

Fig. 6. Fluorine abundances of our O-rich stars sample as a function of the $\mathrm{C} / \mathrm{O}$ ratio. The solid line corresponds to the reference theoretical model, while the dashed line represents a model for which the initial $[\mathrm{F} / \mathrm{Fe}]$ is -0.71 instead of 0 .

obtaining the $\mathrm{F}$ abundance in stars. Various hypotheses on the origin of this element have been discussed in the literature (see Schuler et al. 2007, for a recent overview). In particular, from a correlation of the carbon abundance with the $\mathrm{F}$ abundance, Jorissen et al. (1992) showed that nucleosynthesis in AGB stars is one likely path for the production of this element. Schuler et al. (2007) come to a similar result from $\mathrm{F}$ abundance measurements of a carbon-enhanced, metal-poor star. A positive correlation between $\mathrm{F}$ and $\mathrm{C} / \mathrm{O}$ has also been acknowledged in a planetary nebula (Zhang \& Liu 2005). Our data of NGC 1846 AGB stars clearly support the production and dredge up of $\mathrm{F}$ during the AGB evolution. The two lines in the figure represents the predictions of the reference model. The only difference between the two lines is in the initial fluorine abundance. We have assumed $[\mathrm{F} / \mathrm{Fe}]=0$ and $[\mathrm{F} / \mathrm{Fe}]=-0.71$ at the beginning of the evolutionary sequence, for the solid and the dashed lines, respectively. The lower value corresponds to the one of the less evolved AGB star in our sample (H39). Note that Cunha et al. (2003) report similar fluorine underabundances in LMC giants. Our models predict a negligible variation in the surface $\mathrm{F}$ abundance after the first dredge up. It should be noted that only an "extreme" extra mixing during the RGB may induce a sizeable F depletion (see e.g. Denissenkov et al. 2006). In contrast, during the AGB fluorine is produced by $\alpha$ captures on ${ }^{15} \mathrm{~N}$ in the convective zone generated by a thermal pulse and, later on, dredged up. Part of the ${ }^{15} \mathrm{~N}$ seeds are in the ashes of the CNO burning (about $50 \%$ ), while the remaining part is synthesized in the ${ }^{13} \mathrm{C}$ pocket. In both cases, ${ }^{15} \mathrm{~N}$ is released by the ${ }^{18} \mathrm{O}(p, \alpha){ }^{15} \mathrm{~N}$ reaction (for more details see Cristallo et al. 2006). However, even if models account for a substantial AGB production, we confirm the previous claim by Jorissen et al. (1992) that the theoretical predictions underestimate the fluorine overabundance observed in evolved AGB stars. A possible solution may be a more efficient production of ${ }^{15} \mathrm{~N}$ in the He-rich inter-shell zone. Perhaps, an additional and different source of fluorine should be sought. We have to note, of course, that, as the fluorine abundance in the NGC 1846 stars has been derived from only one line blend, a systematic error cannot be excluded. 


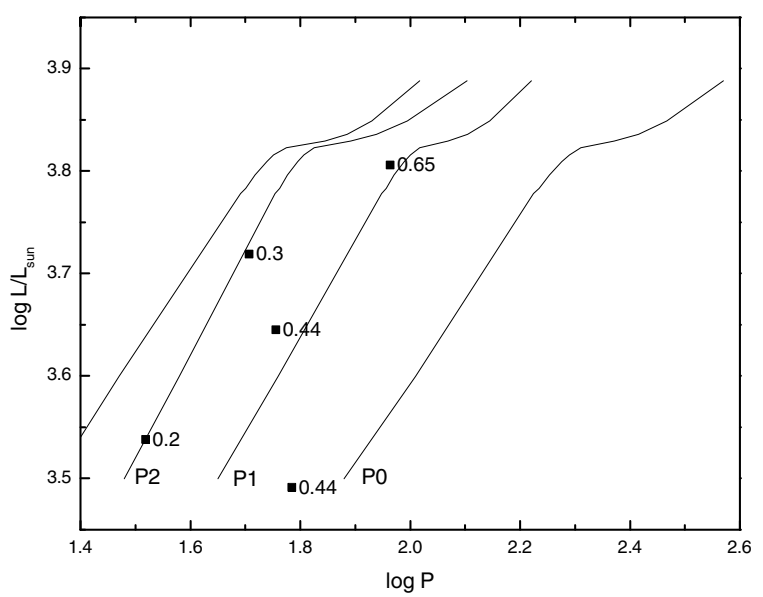

Fig. 7. The $\log \left(L / L_{\odot}\right)-\log P$ diagram for NGC 1846 adapted from Lebzelter \& Wood (2007). Pulsation models for fundamental mode and the first three overtone modes are shown together with five O-rich stars of our sample.

\subsection{Dredge up and pulsation properties}

The detailed variability study on the AGB stars in NGC 1846 undertaken in Lebzelter \& Wood (2007) allows us to investigate the observed star-to-star differences in the $\mathrm{C} / \mathrm{O}$ ratio in light of the pulsational properties of these stars. In Fig. 7 we show the location of our sample stars in the P-L-diagram of the cluster. Data are taken from Lebzelter \& Wood. LE9 could not be plotted due to the lack of any detected periodicity in its small-amplitude light variability. The other stars can be easily attributed to a pulsation mode: LE13 and LE16 are first overtone pulsators, while LE8 and $\mathrm{H} 39$ pulsate in the second overtone. Long secondary periods beyond the fundamental mode period have been found in the light curves of several of these stars, but no shorter secondary periods. As already pointed out in Lebzelter \& Wood (2007), LE17 cannot be attributed unequivocally to any pulsation sequence.

Stars located on the first overtone sequence show a higher $\mathrm{C} / \mathrm{O}$ ratio than objects on the second overtone sequence (Fig. 7). As can be seen this cannot be attributed to a simple difference in luminosity as the two groups clearly overlap. This is further illustrated in Fig. 8 where we marked the sample stars in the color-magnitude diagram according to their pulsation mode. For LE9 $(\mathrm{C} / \mathrm{O}=0.2)$, no periodic change was found by Lebzelter \& Wood (2007). Assuming that it would have been more likely to find a first overtone pulsation than a shorter period higher overtone pulsation, we attributed this star to the group of higher overtone pulsators. These findings suggest that in addition to a relation between the $\mathrm{C} / \mathrm{O}$ ratio and the luminosity, seems to exist some dependency of this ratio on the pulsation mode. At this point it is not clear how to explain the existence of such a relation. As mentioned above, all sample stars are on the AGB, we can exclude an RGB contamination. From the difference in the $\mathrm{C} / \mathrm{O}$ ratio, we can assume that the sample stars are in at least three different thermal pulse cycles. Both from our models and from the literature (e.g. Vassiliadis \& Wood 1993), we would expect that the stars become cooler from cycle to cycle. As the two C enhanced stars LE16 and LE13 would form a sequence at higher temperatures than the other stars, it can be excluded that a difference in the thermal pulse cycle is responsible for the observed differences in pulsation mode and temperature.

A difference in pulsation mode between two stars of the same luminosity requires a difference in some other global characteristic of the star. This could be a difference in mass.

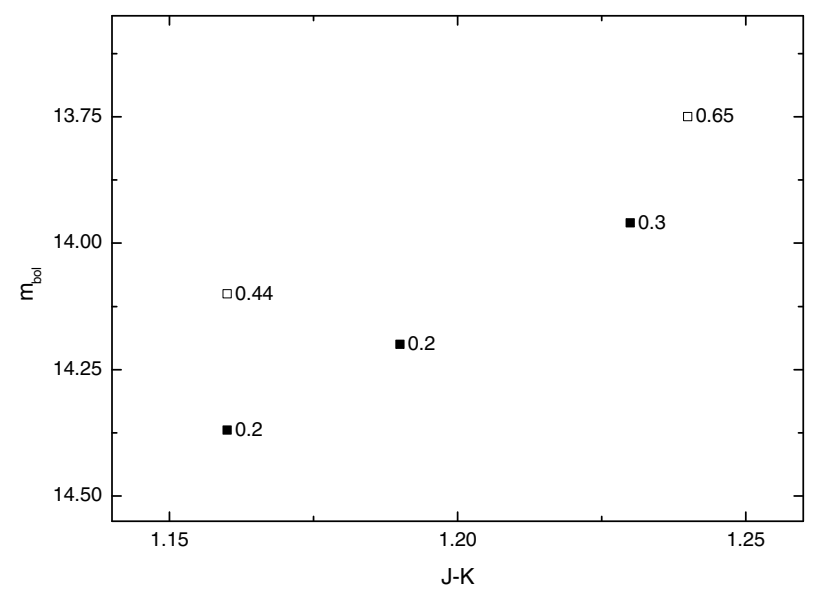

Fig. 8. Location of the O-rich stars in a color-magnitude diagram with the pulsation mode of each object indicated. Open boxes denote first overtone pulsators, while filled boxes mark stars with second overtone pulsation. The star LE17 has been excluded.

Mackey \& Broby Nielsen (2007) report indications for two stellar populations in NGC 1846 separated in age by about 300 Myr. At a given time we may then find AGB stars of different masses at the same time on the AGB. Indeed such an age or mass difference could easily account for the observed color difference (Fig. 8). This would also be compatible with the observed $\mathrm{C} / \mathrm{O}$ ratios as the more massive stars may show a more efficient dredge up. Furthermore, the starting abundances of the two populations may be different, which could enhance the observed difference in $\mathrm{C} / \mathrm{O}$ and ${ }^{12} \mathrm{C} /{ }^{13} \mathrm{C}$.

It has to be stressed that, even in the case of a single stellar population, a scatter in the abundances of $\mathrm{C}$ and $\mathrm{O}$ on the main sequence and after the first dredge up may be present. Such an abundance scatter has been found in various clusters.

\subsection{The S-type stars}

An interesting case is LE13. This star has been classified as spectral type S3/3 by Lloyd Evans (1983). Indeed the ZrO band head at $6345 \AA$ is clearly visible in the spectrum presented by him (see his Fig. 1). According to its spectral classification, TiObands and $\mathrm{ZrO}$ bands should be of similar strength (Keenan \& Boeshaar 1980). YO should occur, but is not covered by the spectra shown by Lloyd Evans. As expected for a star with S-type spectral characteristics, the $\mathrm{C} / \mathrm{O}$ ratio is clearly enhanced in LE13 with a value between 0.6 and 0.7. In Fig. 9 we compare the observed spectrum with our model for both cases $(\mathrm{C} / \mathrm{O}=0.6$ and $\mathrm{C} / \mathrm{O}=0.7)$. The model obviously fits the observations quite well. As mentioned above, this model was calculated with an increased microturbulent velocity to allow for a reasonable fit of the observed line profiles. A change in the $\mathrm{C} / \mathrm{O}$ ratio to 0.7 gives a similar fit, but a further increase in the $\mathrm{C} / \mathrm{O}$ ratio gets problematic as the $\mathrm{CN}$ lines, especially the one at $15567 \AA$, become too strong. Compensating for this with a higher temperature would give a bad fit of the $\mathrm{OH}$ line close to $15570 \AA$ and too strong a $\mathrm{CO}$ band head. Similarly, changes in $\log g$ cannot compensate for a $\mathrm{C} / \mathrm{O}$ value close to one. LE13 is thus an S-type star with C/O clearly below 1 .

Lloyd Evans (1983) also classified a second star in this cluster as of spectral type S3/1, namely LE8. LE8 has an even lower $\mathrm{C} / \mathrm{O}$ ratio of only about 0.3 . Direct comparison with the spectra of LE9 and H39 does indicate that the star has an increased 


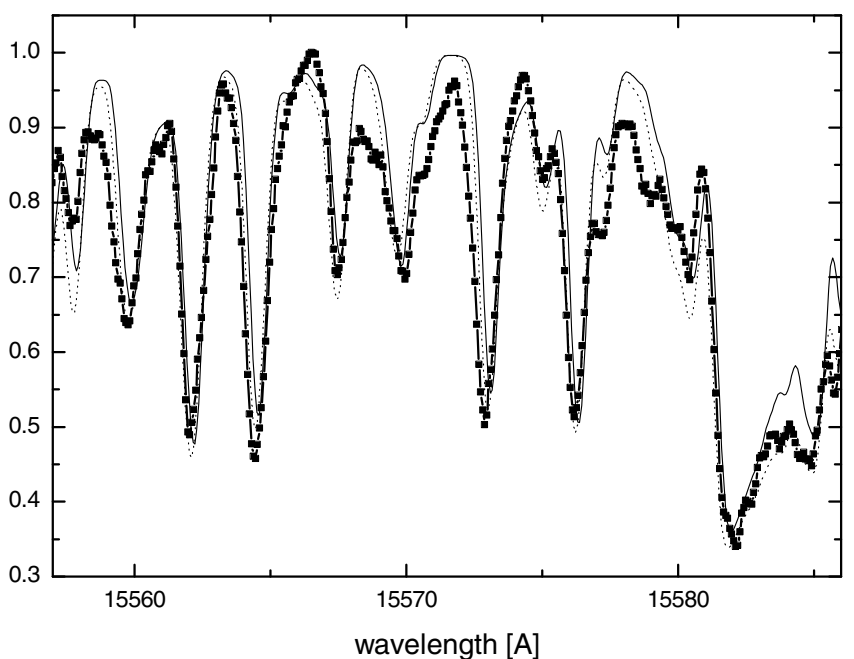

Fig. 9. $H$ band spectrum of LE13 compared with models of $3600 \mathrm{~K}$, $\log g=0, \mathrm{C} / \mathrm{O}=0.6$ (solid line) and 0.7 (dotted line), respectively.

$\mathrm{C} / \mathrm{O}$ ratio, but it is far below the expectations for an S-star. Unfortunately, Lloyd Evans (1983) presented no visual spectrum of this star.

A C/O ratio of only 0.65 for an S-type star is in agreement with the oxygen overabundance in the Magellanic Cloud discussed above. If this the case for NGC 1846, and the comparison of the other stars with the expectations from the model suggests this, then we would require more dredge up events to produce a $\mathrm{C} / \mathrm{O}$ ratio close to one than in the case of a solar abundance pattern. In other words, the abundance of s-process enriched material on the surface may be high enough to see e.g. $\mathrm{ZrO}$ bands, but the amount of $\mathrm{C}$ dredged up does not yet equal the amount of $\mathrm{O}$ due to the high starting value of the $\mathrm{O}$ abundance. Comparing the $\mathrm{C} / \mathrm{O}$ ratio of $\mathrm{LE} 13$ and LE8, we would expect to see less $\mathrm{ZrO}$ in LE8, which indeed agrees with the spectral classes from Lloyd Evans (1983).

Piccirillo (1980) pointed out that, only for stars with $T<$ $3000 \mathrm{~K}$, would we expect that the occurrence of $\mathrm{ZrO}$ bands correlates with a $\mathrm{C} / \mathrm{O}$ close to one. For hotter stars, an increased abundance of $\mathrm{Zr}$ is the main reason for a visibility of $\mathrm{ZrO}$ in the spectrum. As the stars in our sample all have temperatures above $3000 \mathrm{~K}$, the occurrence of $\mathrm{ZrO}$ bands is not surprising. We think that a combination of this effect and an enhancement of $\mathrm{O}$ in the pre-AGB composition of the star explains the observed C/O ratio. In this context we also note that Smith \& Lambert (1990) report a $\mathrm{C} / \mathrm{O}$ for the two intrinsic (i.e. AGB, see Jorissen et al. 1993) S-stars NQ Pup and V679 Oph of 0.29 and 0.75, respectively. Thus the $\mathrm{C} / \mathrm{O}$ values we found for the two S-type stars in NGC 1846 look realistic.

\subsection{The C-stars}

The study of the C-stars in this cluster was hampered by these difficulties deriving basic parameters and abundances. Still a few points can be noticed. The $\mathrm{C} / \mathrm{O}$ ratio we find is rather high. There is quite a gap in our sample between the highest $\mathrm{C} / \mathrm{O}$ ratio of an O-rich star (LE13, 0.65) and the lowest value of a C-rich star (LE11, 1.7). There are still two candidates in the cluster, LE12 and LW2 (see Lebzelter \& Wood 2007), which may fill this gap, but unfortunately we have no data for these stars. The value is also high compared to the values found by Lambert et al. (1986; see also Smith \& Lambert 1990) for field stars, where the maximum $\mathrm{C} / \mathrm{O}$ is 1.6. Such an occurrence is not surprising, however, since the oxygen content is lower in NGC 1846, even considering $[\mathrm{O} / \mathrm{Fe}]=0.2$, when compared to field stars. From the abundance of $\mathrm{HCN}$ and $\mathrm{C}_{2} \mathrm{H}_{2}$, Matsuura et al.(2006) find that the $\mathrm{C} / \mathrm{O}$ ratio in their sample of $\mathrm{LMC}$ carbon stars should be higher than 1.7, which would agree with our findings.

Of particular interest is the carbon isotopic ratio derived for the 2 carbon stars in our sample. Although the $\mathrm{C} / \mathrm{O}$ ratio increases, the ${ }^{12} \mathrm{C} /{ }^{13} \mathrm{C}$ remains close to the highest value found for the O-rich stars in this cluster. Basing on the only $2 \mathrm{C}$ stars in the sample, we can tentatively conclude that some saturation level exists around 60 for the carbon isotopic ratio. In contrast to this figure, the evolutionary models predict a continuous increase in the ${ }^{12} \mathrm{C} /{ }^{13} \mathrm{C}$ ratio, as due to the dredge up of primary ${ }^{12} \mathrm{C}$. Indeed, for $\mathrm{C} / \mathrm{O}>1$, our reference model predicts values definitely higher than 100 . Our finding confirms what has already been observed in galactic C-stars (Smith \& Lambert 1990; Abia et al. 2001), which shows a clustering between 50 and 70 .

The most obvious explanation for this observational pattern is the occurrence of a mixing process able to bridge the radiative gap between the cool bottom of the convective envelope and the hot H-burning zone. A similar process seems to explain some abundance anomalies in red giants stars (see, e.g., Charbonnel 1995). It is usually referred to as extra or deep mixing. More recently, Nollett et al. (2003) have also proposed a deep circulation in low mass AGB stars as a solution to the puzzling isotopic anomalies found in some pre-solar grains. They named this circulation the cool bottom process. In spite of all the observational evidence, a common consensus on the physical mechanism driving this mixing has not been established yet (e.g. rotational induced instabilities, magnetically induced circulation, gravity wave, or thermohaline mixing - see e.g. Busso 2007, for a recent review).

The scenario emerging from the evolutionary sequence of ${ }^{12} \mathrm{C} /{ }^{13} \mathrm{C}$ versus C/O in NGC 1846 is that of a moderate deep mixing, affecting the ${ }^{13} \mathrm{C}$ surface abundance in the late part of the AGB only, when stars become C-rich. Indeed, while the abundance derived from O-rich stars in NGC 1846 are all compatible with the prediction of models with no extra mixing, the ${ }^{12} \mathrm{C} /{ }^{13} \mathrm{C}$ ratios we find for the two $\mathrm{C}$ stars indicate that their envelope material could have been exposed to a temperature on the order of $30-40 \times 10^{6} \mathrm{~K}$. At that temperature and on an AGB timescale, ${ }^{12} \mathrm{C}$ is partially converted into ${ }^{13} \mathrm{C}$, but only marginally into ${ }^{14} \mathrm{~N}$.

From the models, we find that, during the AGB the bottom of the convective envelope, whose temperature never exceeds $4 \times 10^{6} \mathrm{~K}$, moves closer to the H-burning zone. Just before the first thermal pulse, a region as large as $2.2 \times 10^{-3} M_{\odot}$ separates the innermost convectively unstable layer from the shell where the temperature is $40 \times 10^{6} \mathrm{~K}$, but before the 10 th $\mathrm{TP}$, this region is reduced to $4 \times 10^{-4} M_{\odot}$. Such an occurrence suggests that the effect of a possible extra mixing should be stronger in the late part of the AGB than at the beginning.

To simulate such a scenario, we calculated an additional model with the same input parameters as the reference model, but artificially including an extra-mixing. We assume that such an extra-mixing is confined within a region as large as $10^{-3} M_{\odot}$ below the convective envelope, but only if the temperature is lower than a maximum value $\left(T_{\mathrm{lim}}\right)$. The average convective velocity is fixed to $100 \mathrm{~cm} \mathrm{~s}^{-13}$. The most important parameter is, here, the value of the maximum temperature, as shown in

\footnotetext{
${ }^{3}$ This is a slow mixing, when compared to the typical convective velocity in the envelope of an AGB star, which is of the order of $10^{5} \mathrm{~cm} / \mathrm{s}$. We have verified, however, that the $\mathrm{C} / \mathrm{O}$ and ${ }^{12} \mathrm{C} /{ }^{13} \mathrm{C}$ ratios are only marginally affected by the choice of this parameter.
} 


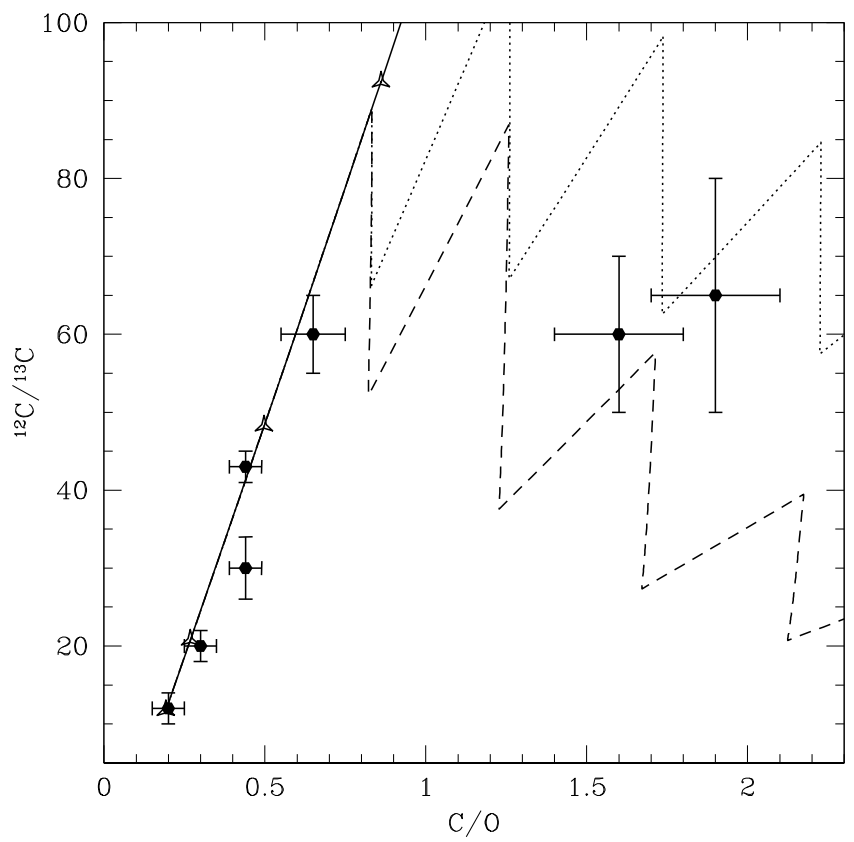

Fig. 10. $\mathrm{C} / \mathrm{O}$ versus ${ }^{12} \mathrm{C} /{ }^{13} \mathrm{C}$ for all the sampled stars. Predicted values from models with and without extra-mixing are shown. Solid line refers to our reference model, while the dotted and dashed ones refer to models with $T_{\text {lim }}=35 \times 10^{6} \mathrm{~K}$ and $T_{\text {lim }}=40 \times 10^{6} \mathrm{~K}$, respectively (see text for details).

Fig. 10, where we reported the ${ }^{12} \mathrm{C} /{ }^{13} \mathrm{C}$ versus $\mathrm{C} / \mathrm{O}$ relations obtained for $T_{\lim }=35$ and $40\left(10^{6} \mathrm{~K}\right)$.

This phenomenological model is useful for inferring the extension of the region affected by the supposed extra-mixing and its maximum temperature, but a more physical description of the mechanism supporting this phenomenon is needed to derive more quantitative results. Either way, we stress that the ${ }^{12} \mathrm{C} /{ }^{13} \mathrm{C}$ versus $\mathrm{C} / \mathrm{O}$ sequences for intermediate age clusters are the best way to understand and to constrain the deep mixing in AGB stars.

\section{Conclusions}

We derived $\mathrm{C} / \mathrm{O}$ ratios and ${ }^{12} \mathrm{C} /{ }^{13} \mathrm{C}$ ratios for the first time for a sample of AGB stars above the dredge-up mass limit within one stellar cluster. In this way we tested how the various third dredge-up events change the surface abundances in a homogeneous sample of AGB stars. Oxygen-rich stars with clear indications of a third dredge-up could be identified from enhanced values of these two quantities, which lie between 0.2 and 0.65 for $\mathrm{C} / \mathrm{O}$ and 12 to 60 for ${ }^{12} \mathrm{C} /{ }^{13} \mathrm{C}$. The cluster also contains a few $\mathrm{C}$-stars, but only rather uncertain values for the $\mathrm{C} / \mathrm{O}$ ratio could be derived. A more detailed analysis for two C-stars was possible leading to $\mathrm{C} / \mathrm{O}=1.7$ and 1.9 and ${ }^{12} \mathrm{C} /{ }^{13} \mathrm{C}=60$ and 65 .

As expected, the $\mathrm{C} / \mathrm{O}$ ratio is correlated with the ${ }^{12} \mathrm{C} /{ }^{13} \mathrm{C}$ ratio. We find good agreement with model predictions for both ratios assuming an oxygen overabundance of +0.2 dex in the cluster. The measured $\mathrm{C} / \mathrm{O}$ ratios are in reasonable agreement with the predicted interpulse values. This is the first direct test of the models concerning dredge up before the stars become $\mathrm{C}$-stars.

A possible correlation between $\mathrm{C} / \mathrm{O}$ ratio and pulsation mode was found. The difference in pulsation mode may also help to explain the observed scatter in $\mathrm{C} / \mathrm{O}$ along the AGB of the cluster, although no final understanding of this relation could be achieved. The findings may be related to the existence of two populations within the cluster with a small difference in age. Further investigations of the populations in this cluster are needed.

An increase in the fluorine abundance along the AGB has been found. This confirms nucleosynthesis in AGB stars as one of the origins of this element.

Two stars in the cluster were classified as having spectral type $\mathrm{S}$ in the past. We find a $\mathrm{C} / \mathrm{O}$ of 0.65 and 0.3 for these objects. While the $\mathrm{C} / \mathrm{O}$ ratio is thus clearly less than 1 , we argue that the comparably high temperature of the stars allows for S-type characteristics at this $\mathrm{C} / \mathrm{O}$.

The ${ }^{12} \mathrm{C} /{ }^{13} \mathrm{C}$ ratio found in the $\mathrm{C}$-stars cannot be explained with standard models for the third dredge up. They indicate the need for a moderate extra-mixing, affecting the late part of the AGB evolution.

Acknowledgements. This work was supported by the Austrian FWF under project number P18171-N02. MTL has been supported by the Austrian Academy of Sciences (DOC programme). Based on observations obtained at the Gemini Observatory, which is operated by the Association of Universities for Research in Astronomy, Inc., under a cooperative agreement with the NSF on behalf of the Gemini partnership: the National Science Foundation (United States), the Science and Technology Facilities Council (United Kingdom), the National Research Council (Canada), CONICYT (Chile), the Australian Research Council (Australia), CNPq (Brazil), and SECYT (Argentina). The observations were obtained with the Phoenix infrared spectrograph, which was developed and is operated by the National Optical Astronomy Observatory. The spectra were obtained as part of program GS-2005B-C-7.

\section{References}

Abia, C., Busso, M., Gallino, R., et al. 2001, ApJ, 559, 1117

Anders, E., \& Grevesse, N. 1989, Geochim. Cosmochim. Acta, 53, 197 Aringer, B. 2000, Ph.D. Thesis, University of Vienna

Aringer, B., Kerschbaum, F., \& Jørgensen, U. G. 2002, A\&A, 395, 915 Busso, M. 2007, ASPC, 378, 26

Busso, M., Gallino, R., \& Wasserburg, J. 1999, ARA\&A, 37, 239

Busso, M., Gallino, R., Lambert, D. L., Travaglio, C., \& Smith, V. V. 2001, ApJ, 557,802

Charbonnel, C. 1995, ApJ, 453, 41

Chieffi, A., Limongi, M., \& Straniero, O. 1998, ApJ, 502, 737

Cristallo, S., Gallino, R., Straniero, O., Piersanti, L., \& Domínguez, I. 2006, Mem. Soc. Astron. Italiana, 77, 774

Cristallo, S., Straniero, O., Lederer, M. T., \& Aringer, B. 2007, ApJ, 667, 489

Cunha, K., Smith, V. V., Lambert, D. L., \& Hinkle, K. H. 2003, AJ, 126, 1305

Davis, S., Wallace, L., Brault, J., \& Engleman, R. 2005, The CN spectrum from the infrared to the ultraviolet. NSO Technical Report

Denissenkov, P. A., Pinsonneault, M., \& Terndrup, D. M. ApJ, 651, 438

Freeman, K. C., Illingworth, G., \& Oemler, A. 1983, ApJ, 272, 488

Frogel, J. A., Mould, J., \& Blanco, V. M. 1990, ApJ, 352, 96

Girardi, L., Chiosi, C., Bertelli, G., \& Bressan, A. 1995, A\&A, 298, 87

Gratton, R. G., Sneden, C., Carretta, E., \& Bragaglia, A. 2000, A\&A, 354, 169

Grevesse, N., \& Noels, A. 1993, Phys. Scrip. Vol. T, 47, 133

Grocholski, A. J., Cole, A. A., Sarajedini, A., et al. 2006, AJ, 132, 1630

Gustafsson, B., Bell, R. A., Eriksson, K., \& Nordlund, A. 1975, A\&A, 42, 407

Harris, M. J., Lambert, D. L., Hinkle, K. H., Gustafsson, B., \& Eriksson, K. 1987, ApJ, 316, 294

Herwig, F. 2000, A\&A, 360, 952

Hill, V., François, P., Spite, M., Primas, F., \& Spite, F. 2000, A\&A, 364, L19

Hinkle, K. H., Cuberly, R., Gaughan, N., et al. 1998, Proc. SPIE, 3352, 810

Houdashelt, M. L., Bell, R. A., \& Sweigart, A. V. 2000a, AJ, 119, 1448

Houdashelt, M. L., Bell, R. A., Sweigart, A. V., \& Wing, R. F. 2000b, AJ, 119, 1424

Iben, I. Jr., \& Renzini, A. 1983, ARA\&A, 21, 271

Jørgensen, U. G., Johnson, H. R., \& Nordlund, A. 1992, A\&A, 261, 263

Jorissen, A., Smith, V. V., \& Lambert, D. L. 1992 A\&A, 261164

Jorissen, A., Frayer, D. T., Johnson, H. R., Mayor, M., \& Smith, V. V. 1993, A\&A, 271, 463

Karakas, A., \& Lattanzio, J. 2007, PASA, 24, 103

Keenan, P. C., \& Boeshaar, P. C. 1980, ApJS, 43, 379

Kupka, F. G., Ryabchikova, T. A., Piskunov, N. E., Stempels, H. C., \& Weiss, W. W. 2000, BaltA, 9, 590

Lambert, D. L., Gustafsson, B., Eriksson, K., \& Hinkle, K. H. 1986, ApJS, 62, 373 
Lebzelter, T. \& Hron, J. 2003, A\& A, 411, 533

Lebzelter, T., \& Wood, P. R. 2007, A\&A, 475, 643

Lederer, M. T., \& Aringer, B. 2007, AIP Conf. Proc. [arXiv:0712.2772v2], submitted

Lloyd Evans, T. 1980, MNRAS, 193, 87

Lloyd Evans, T. 1983, MNRAS, 204, 985

Lodders, L. 2003, ApJ, 591, 1220

Loidl, R., Lançon, A., \& Jørgensen, U. G. 2001, A\&A, 371, 1065

Mackey, A. D., \& Broby Nielsen, P. 2007, MNRAS, 379, 151

Marigo, P., Girardi, L., \& Bressan, A. 1999, A\&A, 344, 123

Matsuura, M., Wood, P. R., Sloan, G. C., et al. 2006, MNRAS, 371, 415

Nollett, K. M., Busso, M., \& Wasserburg, G. J. 2003, ApJ, 582, 1036

Olszewski, E. W., Schommer, R. A., Suntzeff, N., \& Harris, H. C. 1991, AJ, 101, 515

Piccirillo, J. 1980, MNRAS, 190, 441

Piersanti, L., Straniero, O., \& Cristallo, S. 2007, A\&A, 462, 1051

Schommer, R. A., Olszewski, E. W., Suntzeff, N., \& Harris, H. C. 1992, AJ, 103, 447
Schuler, S. C., Cunha, K., Smith, V. V., et al. 2007, ApJ, 667, L81

Smith, V. V., \& Lambert, D. L. 1990, ApJS, 72, 387

Sneden, C. 1991, in Evolution of Stars: The Photospheric Abundance Connection, ed. G. Michaud, \& A. Tutukov (Dordrecht: Kluwer), IAU Symp., 145,235

Stancliffe, R. J., Izzard, R. G., \& Tout, C. A. 2004, MNRAS, 356, 4

Straniero, O., Chieffi, A., \& Limongi, M., 1997, ApJ, 478, 332

Straniero, O., Domínguez, I., Cristallo, S., \& Gallino, R. 2003, PASA, 20, 389

Straniero, O., Gallino, R., \& Cristallo, S. 2006, Nucl. Phys. A , 777, 311

Tanabé, T., Nishida, S., Nakada, Y., et al. 1997, Ap\&SS, 255, 407

van Eck, S., Goriely, S., Jorissen, A., \& Plez, B. 2001, Nature, 412, 793

Vassiliadis, E., \& Wood, P. R. 1993, ApJ, 413, 641

Windsteig, W., Dorfi, E. A., Höfner, S., Hron, J., \& Kerschbaum, F. 1997, A\&A, 324,617

Wood, P. R., Olivier, E. A., \& Kawaler, S. D. 2004, ApJ, 604, 800

Zhang, Y., \& Liu, X.-W. 2005, ApJ, 631, 61 